\title{
Los derechos humanos en la política exterior de la Unión Europea
}

\author{
Armando Alvares GARCIA JÚNIOR*
}

*Profesor de derecho internacional público, derecho del comercio internacional (grado en derecho), estructura económica mundial, negocios internacionales (grado ADE) y negociación y marketing internacional (MBA ADE) en la Universidad Internacional de la Rioja, España.

armando.alvares@unir.net

RESUMEN: La política exterior de la Unión Europea fomenta la democracia y la protección de los derechos humanos en todo el mundo. Sin embargo, un análisis histórico más rigoroso señala el tortuoso camino de los acuerdos internacionales firmados con los países en desarrollo hasta llegar al estatus actual: convertirse en condición esencial en las relaciones de cooperación internacional.

PALABRAS CLAVE: Derechos humanos, política exterior, Unión Europea, comercio, cooperación, países en desarrollo

\section{Human rights in foreign policy of the European Union}

\begin{abstract}
The foreign policy of the European Union promotes democracy and protection of human rights worldwide. However, a more rigorous historical analysis indicates the tortuous path of international agreements signed with developing countries to reach the current status: becoming an essential condition in international partnerships.
\end{abstract}

KEYWORDS: Human rights, foreign policy, European Union, trade, cooperation, developing countries.

Recebido em: 10/05/2013 - Aprovado em: 10/07/2013 - Disponibilizado em: 15/08/2013

\section{DERECHOS HUMANOS: DESARROLLO EN LA POLÍTICA EXTERIOR DE LA UNIÓN EUROPEA}

La apreciación de los derechos humanos y laborales está estrechamente relacionada con el comercio internacional actual.

La efectiva observancia y aplicación de los convenios de protección de los derechos humanos y normas laborales internacionales, por parte de terceros estados, funciona, en el ámbito de los acuerdos comerciales llevados a cabo por la Unión Europea, como un elemento fundamental en lo que concierne a la concesión de beneficios arancelarios y facilidad de acceso a su mercado.

Desde 1992, la presencia de cláusulas pertinentes a los derechos humanos es constante en los tratados bilaterales de comercio y en los acuerdos de 
cooperación y asociación suscritos por el bloque europeo.

Su inobservancia por los demás países signatarios implica en la revocación de los beneficios contemplados en los regímenes jurídicos plasmados en el sistema de preferencias generalizadas (SPG), un instrumento especialmente relevante de la política comercial común y de ayuda al desarrollo, cuyo objetivo oficial es reducir la pobreza, garantizar los derechos humanos $y$ laborales fundamentales y promover el desarrollo sostenible y la gobernanza en los países en vías de desarrollo, mediante la generación de ingresos adicionales a través de sus exportaciones.

Con la incorporación de esa cláusula de derechos humanos, se logra:

a) difundir la idea de universalización de los derechos humanos;

b) difundir los principios más relevantes para el conjunto de la Unión Europea, como la democracia y el estado de derecho;

c) establecer y consolidar, como afirma Szymanski $(2005)^{1}$, una verdadera coherencia entre las políticas internas y externas del bloque.

La actuación exterior de la Unión Europea conecta, de modo inseparable, el fomento del comercio internacional, el crecimiento económico de los países en desarrollo, la reducción de la pobreza

${ }^{1}$ Szymanski, Marcela; Smith, Michael E. (2005). Coherence and Conditionality in European Foreign Policy. Journal of Common Market Studies, 43, 1, 184-187. de sus respectivas poblaciones y la protección efectiva de los derechos humanos.

El otorgamiento de facilidades comerciales de naturaleza esencialmente arancelaria, por parte de la Unión Europea, está vinculado al objetivo institucional de promover una adecuada inserción de los países en desarrollo en el mercado internacional, aunque existan diferencias importantes en relación a su grado de apertura e inserción.

Así, por ejemplo, el acuerdo firmado entre la Comunidad Europea y México, en 1997, ha permitido reducir el excesivo peso de los Estados Unidos en el mercado de ese país.

Evidentemente, el hecho de que México integre, juntamente con Canadá y Estados Unidos, el área de libre comercio de América del Norte, plasmado en el tratado multilateral conocido como NAFTA (North American Free Trade Agreement), no ha permitido la reducción del peso relativo en el grado y en la intensidad deseada por la Unión Europea bajo la perspectiva de sus intereses geoeconómicos.

Lo que importa considerar es que la cláusula de derechos humanos está presente en diversos acuerdos celebrados por el bloque europeo. Así, entre otros, podemos mencionar los acuerdos con Sudáfrica, con los países de la Asociación Europea de Libre Comercio (AELC), con Turquía, Israel, Siria, Líbano, Autoridad Palestina, Rusia, India, Pakistán, Vietnam, Camboya, Bangladesh, Chile, Colombia 
y Perú, además de constituir un elemento relevante en el ámbito de la Unión para el Mediterráneo o en los acuerdos negociados con el Mercosur.

El actual rol representado por la protección de los derechos humanos en los acuerdos internacionales de cooperación y comercio en que participa la Unión Europea no puede ser adecuadamente explicado sin la comprensión de su desarrollo histórico en relación a las antiguas colonias europeas.

Relativamente a esos países, el bloque europeo tiene firmado, desde el año 2000, el Acuerdo de Cotonou, en que promueve el intercambio comercial y de asistencia con 78 estados de África, del Caribe y del Pacífico (los denominados países ACP).

Ese acuerdo ha reemplazado a la Convención de Lomé de los años setenta (aunque la Convención Lomé IV Revisada era del período 1995-2000) que, por su vez, ha sustituido a las convenciones de Yaundé I (1963-1969) y Yaundé II (1969-1975), previstas inicialmente para atender a las relaciones entre el bloque europeo y dieciocho ex colonias francesas y belgas en África. Su fundamento jurídico original se encontraba en el Fondo Europeo de Desarrollo, creado por el Tratado de Roma en 1957.

Aunque sea su base jurídica histórica, los Acuerdos de Yaundé no propiciaban efectos jurídicos concretos en relación a la protección de los derechos humanos que, además, eran mencionados solamente de una forma muy genérica.
Según Bartel $(2005)^{2}$, la generalidad o hasta mismo la ausencia de cláusulas pertinentes a la protección de los derechos humanos en esos acuerdos se debía, esencialmente, a la mentalidad predominante en esa época de que era preciso previamente lograr un suficiente nivel de desarrollo económico para, posteriormente, con el desarrollo social subsecuente, alcanzar un adecuado nivel de protección de los derechos humanos.

Con la incorporación del Reino Unido al bloque europeo, en 1 de enero de 1973, esa superficial alusión a la protección de los derechos humanos ha tenido que ser repensada, puesto que las excolonias británicas señalaban un progresivo empeoramiento de la situación de los derechos humanos entre su población, que ya no contaba con la influencia del poder colonial británico, capaz de garantizar un nivel aceptable de protección.

El resultado ha sido la elaboración del Acuerdo Lomé I (1975) que, sin embargo, tampoco ha logrado modificar sustancialmente la forma como la Comunidad Europea comprendía el tema de los derechos humanos: un tema de naturaleza esencialmente doméstica. Por esa razón, ante la falta de un acuerdo político, el bloque se limitaba a referenciar los principios consagrados en el ámbito de las Naciones Unidas.

Solamente con la dictadura de Idi Amin Dada en Uganda, en 1977, caracterizada por la grave y sistemática violación de

\footnotetext{
${ }^{2}$ Bartel, Lorand. (2005). Human Rights and Democracy Clauses in the EU's International Agreements. Subcommittee on Human Rights on Foreign Affairs, European Parliament, EP\&ExPol/B/2006/06.
} 
los derechos humanos y genocidio, ha ocurrido un cambio de la opinión política en el ámbito europeo.

El Acuerdo de Lomé I no contemplaba la posibilidad de suspensión del acuerdo comercial, de modo que la Comunidad Europea ha tenido que seguir cumpliendo las obligaciones contraídas con ese país africano de buena fe, tal como preconizado por la Convención de Viena sobre el Derecho de los Tratados (1969) en su artículo 26.

Sin embargo, informalmente, se ha optado por el retraso intencional de los pagos relativos a la ayuda al desarrollo a Uganda hasta la caída de Idi Amin en el año 1979.

Si por un lado la asfixia económica, representada por la transferencia de solamente el 5\% de los valores previstos en el acuerdo, ha contribuido al derrocamiento de la dictadura de Idi Amin Dada, cuyo gobierno ya estaba tocado por la guerra con Tanzania entre los años 1978-1979, por otro, la imposibilidad formal de revocar el acuerdo ha demostrado la ineficacia del régimen jurídico adoptado por el bloque europeo en sus relaciones internacionales.

Durante las negociaciones del Acuerdo Lomé II, en 1980, los miembros de la Comunidad Europea tampoco tenían una posición unánime sobre la inclusión de los derechos humanos en los acuerdos comerciales.

Además, existía una fuerte resistencia opuesta por los países ACP, que señalaban la contradicción europea representada por sus buenas relaciones con Sudáfrica durante el régimen Apartheid.

Además, paralelamente, muchos de los países que habían logrado la independencia recientemente, consideraban que condicionar la ayuda a la observancia de los derechos humanos era una actitud neocolonialista por parte de las potencias europeas.

Un último argumento era que ese condicionamiento político atentaba contra su soberanía y podía ser interpretado - y de hecho era así que lo comprendían - como una excusa cuyo real propósito era inmiscuirse en sus asuntos internos, algo vedado por la propia Carta de San Francisco, constitutiva de las Naciones Unidas.

Al final, con todos esos elementos divergentes, el Acuerdo Lomé II no ha logrado cambios significativos en lo que concierne a las relaciones comerciales europeas con terceros países.

En 1985, con la adopción del Convenio de Lomé III, los derechos humanos pasan a ser mencionados en el preámbulo del texto. El paso ha sido tímido, puesto que la mención solamente podía servir para orientar al exegeta en la interpretación de los artículos del propio convenio internacional que, sin embargo, no contemplaba la posibilidad de suspensión del acuerdo en caso de violación de esos derechos.

Con el Acuerdo Lomé IV de 1990, se logra incorporar los derechos humanos, por primera vez (artículo $5^{\circ}$ ), como factor esencial para el desarrollo. 
Sin el apoyo y la voluntad de interceder a favor de los países ACP por parte de la colapsada Unión Soviética, la resistencia de esos países, en una situación económica particularmente débil en la época, prácticamente deja de existir, de modo que la uniformidad política europea, en relación al tema de la inclusión de los derechos humanos en sus acuerdos internacionales, pasa a trillar un camino menos espinoso para prosperar.

\section{EL IMPULSO CONCEPTUAL PROPORCIONADO POR LA SITUACIÓN EN HAITI Y LA EX YUGOSLAVIA}

Las violaciones de los derechos humanos ocurridas en Haití y Yugoslavia, en 1991, han posibilitado a la Comunidad Europea ampliar su foco en relación a esos derechos más allá de la cooperación con los países ACP.

Como explica Hipold $(2002)^{3}$, las protestas políticas y el no desarrollo de los tratados existentes como forma de presión, relativamente a la guerra de secesión en Yugoslavia, han sido reemplazadas por la observancia del acuerdo firmado en 1983 con base en la cláusula rebus sic stantibus que, no obstante, no podía proporcionar seguridad jurídica adecuada para otros casos de violación de derechos humanos.

Con el golpe de estado en Haití, en septiembre de 1991, la situación era muy complicada jurídica y políticamente. Las relaciones entre la

\footnotetext{
${ }^{3}$ Hipold, Peter. (2002). Human Rights Clauses in EU/Association Agreements. Vienna: Springer.
}

Comunidad Europea y ese país estaban centradas en el Acuerdo Lomé IV que, como vimos, no contemplaba la posibilidad de suspensión del acuerdo con base en la violación de los derechos humanos.

Así, la Comunidad Europea, ante la dificultad de compatibilizar, por un lado, la falta de previsión relativa a la suspensión del acuerdo, y por otro, las sanciones impuestas por la Organización de los Estados Americanos (OEA), acaba optando por una tercera vía: no seguir las medidas adoptadas por la entidad panamericana y esperar la adopción de una resolución por parte del Consejo de Seguridad de las Naciones Unidas.

En este sentido, con base en la Resolución $\mathrm{n}^{\circ} 841$ del Consejo de Seguridad de la Organización de las Naciones Unidas (1993), se ha editado, en el ámbito europeo, el Reglamento (CEE) n ${ }^{\circ} 1603$ del Consejo, de 24 de junio de 1993, relativo a la imposición de un embargo sobre determinadas operaciones comerciales entre la Comunidad Europea y Haití.

Posteriormente, y a raíz de los progresos realizados en este país para normalizar su situación ante la organización internacional, el Consejo de Seguridad de las Naciones Unidas ha adoptado la Resolución no 861 (1993), por la que se suspendía referido embargo. En el ámbito europeo, la suspensión al embargo fue plasmada en el Reglamento (CEE) 2520/1993 del Consejo, de 13 de septiembre de 1993.

Finalmente, en virtud de un importante retroceso y deterioro de la situación de 
los derechos humanos en ese país, el Consejo de Seguridad de las Naciones Unidas aprueba una nueva Resolución, la de $n^{\circ} 873$ (1993), por la que revoca la anterior suspensión, aunque contempla algunas excepciones al embargo (aprobadas caso por caso por el Comité establecido en el apartado 10 de la Resolución nº 841).

En este contexto se aprueba el Reglamento (CEE) número 3028/93, del Consejo, de 28 de octubre de 1993, por el que se deroga la suspensión del embargo referente a determinados intercambios comerciales entre la Comunidad Europea y Haití, y por el que se modifica el Reglamento (CEE) número 1608/93 del Consejo por el que se imponía ese embargo.

A partir de ese momento, en virtud de la evolución conceptual y normativa asociada a los eventos del año 1991 en Haití y Yugoslavia, la incorporación de una cláusula relativa a la protección de los derechos humanos se convierte en un elemento fundamental en los tratados firmados por la Comunidad Europea.

$\mathrm{Su}$ incorporación y consolidación ha sido, de un momento a otro, rápida y sólida. En el ámbito estrictamente europeo se puede observar que, mientras los acuerdos con Hungría, Polonia y Checoslovaquia, de diciembre de 1991, no contemplan cláusulas relativas a la protección de los derechos humanos, los acuerdos con Lituania, Letonia, Estonia y Albania, de 11 de mayo de 1992 sí que las incorporan.

Desde esa fecha (11 de mayo de 1992) los derechos humanos y los principios democráticos (cláusula de derechos humanos y cláusula democrática) pasan a actuar como elementos condicionantes en los acuerdos comerciales, de cooperación y de asociación celebrados por la Comunidad Europea con terceros estados.

La incorporación de esas cláusulas ha contribuido al establecimiento de un trato teóricamente igualitario en relación a todos aquellos países en desarrollo que mantuviesen relaciones con la Comunidad Europea. Eso, obviamente, ha repercutido en la normalización de la actuación del bloque europeo en su dimensión externa, en lo que se refiere a su política de derechos humanos.

Para optimizar una fórmula que se ha mostrado adecuada al largo del tiempo y evitar polémicas y controversias sobre su contenido, el bloque europeo, en vez de definir lo que se comprende como derechos humanos y limitar su extensión, opta, en los acuerdos comerciales, de cooperación y de asociación que celebra, por señalar expresamente determinados instrumentos internacionales de referencia, ampliables con el tiempo, para incorporar nuevos y relevantes convenios internacionales pertinentes a los derechos humanos, laborales, medioambientales y de gobernanza.

Es interesante observar que el Acuerdo de Cotonou (que ha sucedido a los Acuerdos de Lomé) relativamente a la cooperación prestada con los países $\mathrm{ACP}$, no hace referencia expresa a la Declaración Universal de los Derechos Humanos de 1948, aunque en su 
artículo $9^{\circ}$ mencione varios de esos derechos.

\section{LA PROTECCIÓN DE LOS DERECHOS HUMANOS COMO CONDICIÓN ESENCIAL DE LOS ACUERDOS INTERNACIONALES: LA INFLUENCIA DE BULGARIA}

Dos otras fechas deben ser mencionadas: 1993 y 1995.

Desde 1993, en razón del acuerdo con Bulgaria, las cláusulas de suspensión de los acuerdos en función de la violación de una parte esencial del tratado (derechos humanos, democracia) son prácticamente iguales.

Finalmente, a partir de 1995, la cláusula de protección de los derechos humanos se ha plasmado en una fórmula estandarizada en todos los tratados internacionales suscritos por la Comunidad Europea con los países en desarrollo.

La denominada "cláusula búlgara" (actualmente también se emplea la expresión "cláusula de condicionalidad negativa") se aplica desde entonces a cualquier país en desarrollo interesado en tener acceso privilegiado al mercado europeo. Eso significa que los acuerdos anteriores con Turquía (1963) no contemplan ese tipo de cláusula.

Aunque celebrado en fechas anteriores, los acuerdos con Suiza (1972) o San Marino (1992, meses antes de la consolidación de la "cláusula búlgara") son distintos, en virtud del mayor nivel de desarrollo socio-económico y de respecto a los derechos humanos existentes en estos países.

Además, hay que mencionar la diferencia de enfoque en el sustrato de los acuerdos, en razón de las históricas relaciones internacionales existentes entre ellos y el bloque europeo (algo similar, en este último aspecto, a los acuerdos celebrados posteriormente con las Islas Feroe en 1996 o Andorra en 2004).

La suspensión de los tratados preferenciales con Níger (1999), Liberia (1999), Comoras (1999), Fiyi (2000), Costa de Marfil (2000), Zimbabue (2002), República Centroafricana (2003) y Guinea-Bissau (2004) ha sido motivada por la violación de los derechos humanos y de la "cláusula democrática": a) golpes de estado, b) corrupción desenfrenada y sistemática, c) empeoramiento general de la situación de los derechos humanos de las respectivas poblaciones.

Hay que enfatizar que relativamente a los países ACP, la violación constatada por la sociedad internacional en general y por la Unión Europea, en concreto, era verdaderamente sistemática, no solamente puntual o verificada en situaciones particulares.

Temas relacionados, por ejemplo, con la protección de las minorías (étnicas, lingüísticas o religiosas), igualdad de género, libertad de prensa, libertad de expresión y de manifestación, intrusiones violentas de los cuerpos de seguridad etc. eran recurrentes en las interpelaciones de los parlamentarios europeos ante el Consejo y a la Comisión Europea. 
Aunque la instrumentalización jurídica se haya consolidado, la influencia política del estado violador ejerce una presión poderosa. Así, no han sido consideradas "violaciones" los actos atribuidos a Israel por la actuación de su ejército sobre objetivos palestinos (destrucción de infraestructuras, asesinatos selectivos, arresto domiciliario del fallecido líder de la OLP Yasser Arafat) o las intervenciones del ejército ruso en Chechenia. No obstante, la gravedad e intensidad de las violaciones sistemáticas eran similares aquellas verificadas, por ejemplo, en Zimbabue, con la expropiación de campesinos.

Como elemento fundamental de la acción exterior de la Unión Europea, los derechos humanos están vinculados a la ayuda al desarrollo. Sin embargo, mientras la cooperación interinstitucional de tipo político tiene un carácter esencialmente unidireccional (Unión Europea ayuda, no el contrario), los acuerdos comerciales tienen un carácter bidireccional más evidente.

En relación a los primeros, la opinión pública (y por tanto, la opinión de los electores votantes) tiende a ejercer mayor presión sobre las instituciones europeas con el objetivo de que apliquen, sin demora, la suspensión de la ayuda a aquellos países violadores de los derechos humanos.

En el segundo caso, el peso de la opinión pública es mucho menor, de modo que la Unión Europea, mediante la actuación de sus órganos competentes, puede evaluar políticamente la conveniencia e interés, a corto, medio y largo plazo, de mantener o no el país referenciado en la lista oficial de beneficiarios de las concesiones comerciales en forma de exoneraciones arancelarias.

La decisión relativa a la suspensión de los acuerdos comerciales con fundamento en la violación de los derechos humanos (tomada en el más alto nivel político en el ámbito del Consejo Europeo) está estrechamente relacionada con el grado de susceptibilidad y repercusión que tendrá ese tipo de medida en la esfera de las relaciones internacionales en que la Unión Europea esté involucrada.

Nadie dudaría que adoptar medidas en relación a países como Bangladesh, Burkina Faso, Camerún, Eritrea, Togo, Tanzania o Zimbabue es menos problemático que a países como Brasil, Rusia, India, China, Sudáfrica (BRICS), México, Emiratos Árabes Unidos o cualquier otro actor importante de la sociedad internacional.

Cuanto mayor la relevancia de los países en vías de desarrollo (PVDs) en la escena internacional, menor la posibilidad de que los países miembros de la Unión Europea se pongan de acuerdo en relación a la adopción de medidas en su contra.

Para evitar ese problema, o al menos para minorarlo, la suspensión del acuerdo ha pasado a basarse en criterios estrictamente objetivos (nivel de renta, violación de los convenios pertinentes a los derechos humanos etc.). 
Sin eses criterios, relativamente a países importantes en el escenario internacional, la adopción de medidas sancionadoras (por ejemplo, suspensión de acuerdos internacionales) tendería a convertirse en meras exhortaciones o reclamaciones conjuntas sin mayores implicaciones económicas, políticas y jurídicas.

En este sentido, Fierro $(2003)^{4}$ afirma que el criterio de la violación de los derechos humanos consagrados en las diversas normas internacionales señaladas por la propia Unión Europea asume un papel objetivo, aunque su supervisión y evaluación depende del seguimiento llevado cabo por los órganos competentes señalados en los tratados y en las estructuras correspondientes en el ámbito europeo.

\section{LA NOVEDOSA CONEXIÓN ENTRE DERECHOS HUMANOS Y ACUERDOS COMERCIALES}

Lo que verdaderamente importa considerar, para efectos de la actuación exterior de la Unión Europea, es que, en relación a la protección y al respecto por los derechos humanos, si ha establecido una importantísima y novedosa conexión con los objetivos propios de un acuerdo de naturaleza mercantil.

Esa conexión es realmente innovadora en el ámbito de las relaciones internacionales $y$ ha representado la superación de una relevante disensión

\footnotetext{
${ }^{4}$ Fierro, Elena. (2003). The EU`s Approach to Human Rights Conditionality in Practice. Den Haag: Nijhoff.
}

existente en el seno mismo de la Comisión Europea.

Efectivamente, la propia Comisión tenía serias dudas en introducir cualquier tipo de condicionamiento político en lo que se refería a las ayudas concedidas a los países en desarrollo.

Sustancialmente, como afirmaba Forcada Barona $(2004)^{5}$, predominaba la idea de que los derechos humanos no podían tener un encuadramiento adecuado en el marco de un tratado de naturaleza comercial.

$\mathrm{Si}$ consideramos - como ejemplo paradigmático - que los acuerdos firmados por Estados Unidos de América no contemplan consecuencias directas por la falta de cumplimiento de los estándares laborales adoptados en el ámbito de la Organización Internacional del Trabajo, podemos exaltar aún más esa construcción peculiar verificada en el marco europeo.

Otro elemento contrastante: los acuerdos comerciales firmados por Estados Unidos suelen contemplar la constitución de un órgano conjunto de naturaleza política, cuya decisión se basa en el consenso entre los países involucrados. La función de esa comisión bilateral es evaluar periódicamente el cumplimiento de las normas laborales.

La situación prevista en el sistema de preferencias generalizadas (SPG) europeo es completamente distinta, puesto que los estados se someten

\footnotetext{
${ }^{5}$ Forcada Barona, I. (2004) El condicionamiento político de la ayuda oficial al desarrollo de la UE. Gaceta Jurídica de la UE, 25,287- 302.
} 
voluntariamente al correcto cumplimiento de la cláusula de derechos humanos (así como a la cláusula democrática y de protección medioambiental).

Esa sumisión voluntaria equivale, de cierta forma, a una renuncia implícita al principio de la no intervención en cuestiones internas. Paralelamente, los países en desarrollo aceptan la correspondiente imposición de medidas de carácter sancionador: la suspensión del acuerdo de preferencias arancelarias.

Esas medidas, evidentemente, poseen una repercusión enorme sobre las empresas ubicadas en los países involucrados, aunque en mayor medida sobre las de los países en desarrollo.

Países como Bielorrusia o Birmania (Myanmar) han sido excluidos del sistema general de preferencias por esos motivos: falta de efectividad y bajos estándares de protección de los derechos humanos, en el ámbito de sus respectivos territorios.

En un contexto de reconfiguración de las relaciones geopolíticas y geoeconómicas en nivel mundial, el enfoque de la universalización de los derechos humanos puede proporcionar una ventaja competitiva importante a la Unión Europea, puesto que su estricta observancia y aplicabilidad por parte de los países en desarrollo condicionará el otorgamiento o la retirada de las concesiones comerciales privilegiadas (exoneración total o parcial de derechos arancelarios) y el levantamiento de obstáculos no arancelarios relativos al acceso de bienes de los PVDs a la intrazona.

\section{REFERÊNCIAS BIBLIOGRÁFICAS}

Bartel, Lorand. (2005). Human Rights and Democracy Clauses in the EU's International Agreements. Subcommittee on Human Rights on Foreign Affairs, European Parliament, EP\&ExPol/B/2006/06.

Fierro, Elena. (2003). The EU's Approach to Human Rights Conditionality in Practice. Den Haag: Nijhoff.

Forcada Barona, I. (2004) El condicionamiento político de la ayuda oficial al desarrollo de la UE. Gaceta Jurídica de la UE, 25,287- 302.

Hipold, Peter. (2002). Human Rights Clauses in EU/Association Agreements. Vienna: Springer.

Szymanski, Marcela; Smith, Michael E. (2005). Coherence and Conditionality in European Foreign Policy. Journal of Common Market Studies, 43, 1, 184187. 